\title{
Estrogen Metabolism and Risk of Breast Cancer in Postmenopausal Women
}

Barbara J. Fuhrman, Catherine Schairer, Mitchell H. Gail, Jennifer Boyd-Morin, Xia Xu, Laura Y. Sue, Saundra S. Buys, Claudine Isaacs, Larry K. Keefer, Timothy D. Veenstra, Christine D. Berg, Robert N. Hoover, Regina G. Ziegler

Manuscript received January 19, 2011; revised July 16, 2011; accepted December 2, 2011.

Correspondence to: Barbara J. Fuhrman, PhD, Hormonal and Reproductive Epidemiology Branch, Division of Cancer Epidemiology and Genetics, National Cancer Institute, NIH, 6120 Executive Blvd, Rm 5100, Bethesda, MD 20892 (e-mail:fuhrmanb@mail.nih.gov).

Background Estrogens are recognized causal factors in breast cancer. Interindividual variation in estrogen metabolism may also influence the risk of breast cancer and could provide clues to mechanisms of breast carcinogenesis. Longstanding hypotheses about how estrogen metabolism might influence breast cancer have not been adequately evaluated in epidemiological studies because of the lack of accurate, reproducible, and high-throughput assays for estrogen metabolites.

Methods We conducted a prospective case-control study nested within the Prostate, Lung, Colorectal, and Ovarian Cancer Screening Trial (PLCO). Participants included 277 women who developed invasive breast cancer (case subjects) and 423 matched control subjects; at PLCO baseline, all subjects were aged 55-74 years, postmenopausal and not using hormone therapy, and provided a blood sample. Liquid chromatography-tandem mass spectrometry was used to measure serum concentrations of 15 estrogens and estrogen metabolites, in unconjugated and conjugated forms, including the parent estrogens, estrone and estradiol, and estrogen metabolites in pathways defined by irreversible hydroxylation at the C-2, C-4, or C-16 positions of the steroid ring. We calculated hazard ratios (HRs) approximating risk in highest vs lowest deciles of individual estrogens and estrogen metabolites, estrogens and estrogen metabolites grouped by metabolic pathways, and metabolic pathway ratios using multivariable Cox proportional hazards models. All statistical tests were two-sided.

Results Nearly all estrogens, estrogen metabolites, and metabolic pathway groups were associated with an increased risk of breast cancer; the serum concentration of unconjugated estradiol was strongly associated with the risk of breast cancer ( $\mathrm{HR}=2.07,95 \%$ confidence interval $[\mathrm{Cl}]=1.19$ to 3.62$)$. No estrogen, estrogen metabolite, or metabolic pathway group remained statistically significantly associated with the risk of breast cancer after adjusting for unconjugated estradiol. The ratio of the 2-hydroxylation pathway to parent estrogens ( $H R=0.66,95 \%$ $\mathrm{Cl}=0.51$ to 0.87 ) and the ratio of 4-hydroxylation pathway catechols to 4-hydroxylation pathway methylated catechols ( $\mathrm{HR}=1.34,95 \% \mathrm{Cl}=1.04$ to 1.72 ) were statistically significantly associated with the risk of breast cancer and remained so after adjustment for unconjugated estradiol.

Conclusions More extensive 2-hydroxylation of parent estrogens is associated with lower risk, and less extensive methylation of potentially genotoxic 4-hydroxylation pathway catechols is associated with higher risk of postmenopausal breast cancer.

J Natl Cancer Inst 2012;104:326-339

Prospective studies of postmenopausal women have consistently demonstrated that higher levels of circulating estradiol, estrone, and estrone sulfate are associated with an increased risk of breast cancer (1). These associations have long been thought to result from mitogenic effects mediated by the estrogen receptor.

It has been hypothesized that estrogen metabolism may also play a role in breast cancer etiology. The parent estrogens (ie, estrone and estradiol) can be irreversibly hydroxylated at the C-2, C-4, or C-16 positions of the steroid ring (Figure 1) to produce estrogen metabolites that differ in their bioavailability to breast tissues (3) and activation of estrogen receptors (4). In addition, catechol estrogens, which are formed by 2- and 4-hydroxylation, can be oxidized to form mutagenic quinones (5-7); this process is prevented by methylation of one of the adjacent hydroxyl groups (8). Estrogens and estrogen metabolites can also be conjugated via sulfation or glucuronidation, each of which modifies bioavailability (9).

Laboratory and clinical studies have suggested several hypotheses about how estrogen metabolism might influence the risk of breast cancer (10-13). However, robust tests of these 
hypotheses in population studies have not been possible due to the limitations of the available assays for measuring concentrations of estrogens and estrogen metabolites. The radioimmunoassays and enzyme-linked immunosorbent assays used in recent decades to measure circulating estradiol and other parent estrogens often displayed low specificity due to antibody cross-reactivity and thus limited accuracy, especially at the low estrogen concentrations characteristic of postmenopausal women. Substantial variations in measures of estrogens and estrogen metabolites have been noted across laboratories, assay protocols, and assay kit manufacturing lots $(14,15)$. Moreover, individual assays have not been developed for many estrogen metabolites. However, the recent development (16) of an accurate, reproducible, and relatively high-throughput liquid chromatography-tandem mass spectrometry (LC/MS/MS) assay to measure concurrently 15 estrogens and estrogen metabolites, in conjugated and unconjugated forms, even at the low concentrations characteristic of postmenopausal women, allows for the first time characterization of this phenotype for epidemiological study.

We used the new assay to compare the estrogen and estrogen metabolite profiles in prospectively collected serum from postmenopausal women with and without breast cancer who reported no use of exogenous hormones at the time of blood collection. Because this is the first large nested case-control study of breast cancer, to our knowledge, to measure the 15 estrogens and estrogen metabolites in circulation, five of which are found in both conjugated and unconjugated forms, we have systematically evaluated the association of each with breast cancer. We also evaluated the associations of total estrogens and estrogen metabolites (the sum of all estrogens and estrogen metabolites), seven metabolic pathway groups, and eight metabolic pathway ratios with the risk of breast cancer. The groups and ratios were based on biochemistry, metabolic pathways, and etiologic hypotheses from laboratory and clinical research. We analyzed estrogens and estrogen metabolites, individually and in total, and the metabolic pathway groups and ratios, both alone and in combination with the serum concentration of unconjugated estradiol, which is acknowledged as a strong predictor of breast cancer risk (1), to examine whether any of these measures of estrogen metabolism contributed additional independent information for predicting the risk of breast cancer. We discuss our results with respect to previous hypotheses about mechanisms of estrogen-mediated carcinogenesis, and we consider the use of estrogen and estrogen metabolite profiles for projecting breast cancer risk.

\section{Methods}

\section{Study Design and Population}

Incident breast cancer case subjects and control subjects were drawn from the 39116 female participants, aged 55-74 years, who were randomly assigned from 1993 through 2001 to the screening arm of the multicenter Prostate, Lung, Colorectal, and Ovarian Cancer Screening Trial (PLCO) (17). At PLCO baseline, participants completed self-administered questionnaires that covered personal characteristics, medical history, and health-related behaviors. Also at baseline, blood samples were drawn at the screening centers and processed within 2 hours according to a standardized

\section{CONTEXT AND CAVEATS}

\section{Prior knowledge}

Serum estrogen concentration is an established predictor of breast cancer risk in postmenopausal women, but whether biomarkers of estrogen metabolism also predict the risk of breast cancer is unclear because of the lack of accurate, reproducible, sensitive, and high-throughput assays for estrogen metabolites.

\section{Study design}

A prospective case-control study nested within the Prostate, Lung, Colorectal, and Ovarian Cancer Screening Trial examining associations between the risk of breast cancer in postmenopausal women not currently using menopausal hormone therapy and serum concentrations of 15 estrogens and estrogen metabolites, in unconjugated and conjugated forms, including the parent estrogens and estrogen metabolites in pathways defined by irreversible hydroxylation at the $\mathrm{C}-2, \mathrm{C}-4$, or $\mathrm{C}-16$ positions of the steroid ring, as measured by liquid chromatography-tandem mass spectrometry.

\section{Contribution}

The serum levels of nearly all estrogens, estrogen metabolites, and metabolic pathway groups were associated with an increased risk of breast cancer, but none of the associations remained statistically significant after adjusting for serum level of unconjugated estradiol. The ratio of 2-hydroxylation pathway estrogen metabolites to parent estrogens and the ratio of 4-hydroxylation pathway catechols to 4-hydroxylation pathway methylated catechols were statistically significantly associated with the risk of breast cancer, even after adjustment for unconjugated estradiol.

\section{Implications}

More extensive 2-hydroxylation of parent estrogens is associated with lower risk of postmenopausal breast cancer, and less extensive methylation of potentially genotoxic 4-hydroxylation pathway catechols is associated with higher risk of postmenopausal breast cancer.

\section{Limitations}

The study population was restricted to postmenopausal women who were not using menopausal hormone therapy at the time of blood collection, which may limit the generalizability of the findings. There was limited inter-individual variation in serum concentrations of estrogens and estrogen metabolites and high correlations among many of the analytes. There was no adjustment for multiple comparisons and thus, some of the findings could be due to chance.

From the Editors

protocol (17). Follow-up was conducted using annual questionnaires that were mailed to the participants. This study was approved by Institutional Review Boards at the US National Cancer Institute and the 10 participating screening centers.

Of the 39116 female participants randomly assigned to the screening arm of the PLCO, 97\% completed a baseline questionnaire and at least one annual follow-up questionnaire. Of these women, 94\% were postmenopausal at baseline; of these women, $47 \%$ reported no use of menopausal hormone therapy at baseline. Among these postmenopausal non-hormone therapy users, 75\% provided blood at baseline and written informed consent for use of the specimen. 


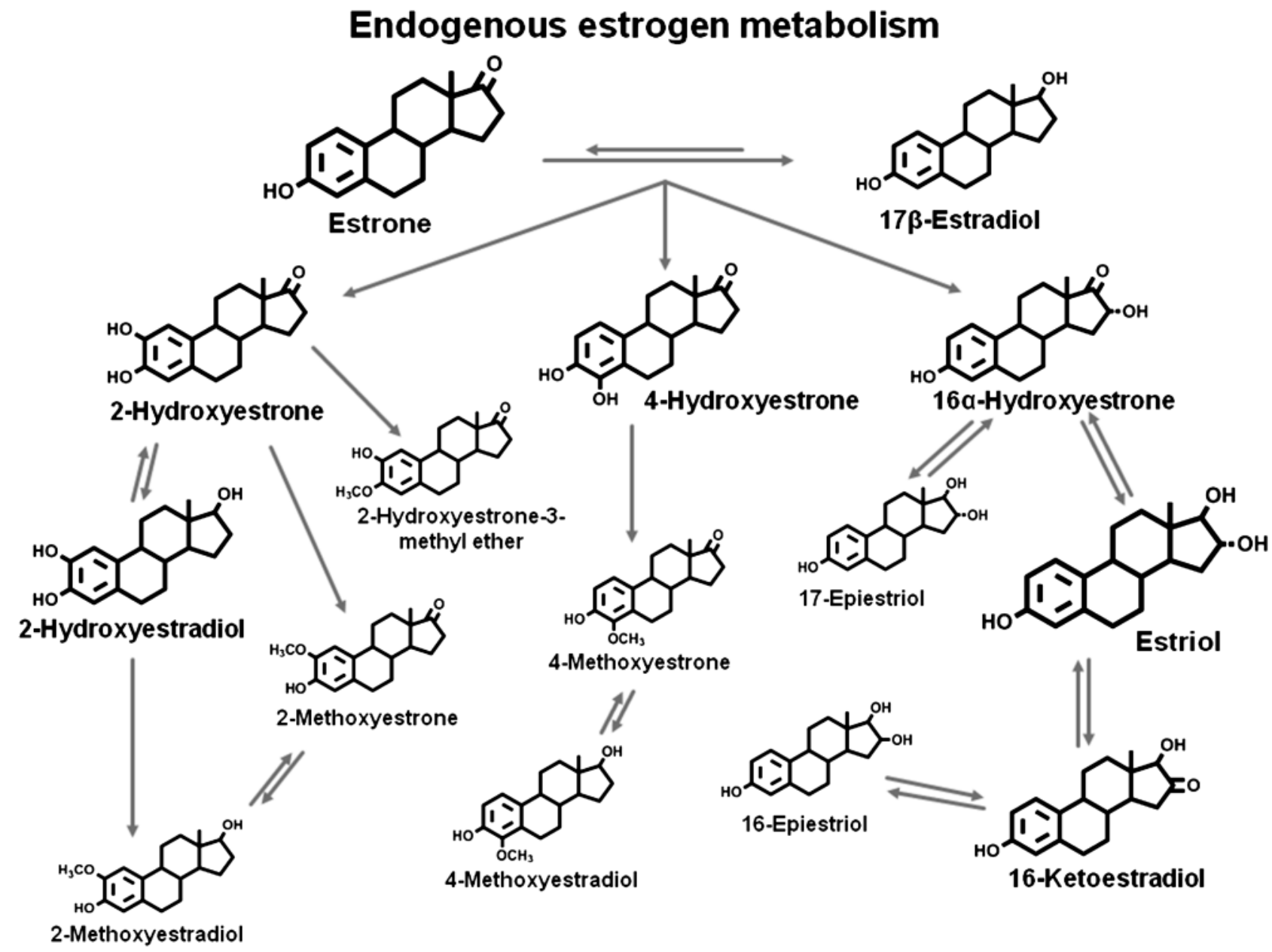

Figure 1. Pathways of estrogen metabolism. Adapted from Ziegler et al. (2) and reproduced with permission from Environmental Health Perspectives. The estrogen metabolites are formed by irreversible hydroxylation of the parent estrogens, estrone and estradiol, at the C-2, C-4, or C-16 positions of the steroid ring. The relative size of the chemical structure indicates the relative concentration of the estrogen or estrogen metabolite in serum of postmenopausal women. The structures are for the unconjugated forms of estrogens and estrogen metabolites.

In the PLCO, incident breast cancers were ascertained primarily via annual questionnaires; incident breast cancers were also identified through state cancer registries, the National Death Index, physician reports, and next-of-kin reports. Of the breast cancers ascertained by these methods, $96.4 \%$ were subsequently confirmed by hospital records (17).

For the initial studies of breast cancer in the PLCO, the cohort of eligible women was defined as all women randomly assigned to the PLCO screening arm who had completed the baseline questionnaire and at least one study update questionnaire, reported no history of breast cancer at baseline, provided blood at baseline or during follow-up, and given written informed consent (16) (Supplementary Table 1, available online). As of June 30, 2005, 1141 incident breast cancers had been ascertained within the study cohort. Control subjects $(\mathrm{n}=1141)$ were randomly selected from women in the study cohort who were alive and free of breast cancer as of June 30, 2005, and were frequency matched to case subjects on age at study entry $(55-59,60-64,65-69,70-74$ years $)$ and period of blood collection (before vs on or after the median collection date, September 30, 1997).

The subjects for this analysis were drawn from this set of 1141 case subjects and 1141 control subjects, as presented in detail in
Supplementary Table 1 (available online). In brief, 440 case subjects and 525 control subjects were postmenopausal and not using hormone therapy at baseline; of these, 424 case subjects and 506 control subjects also had no other cancer, other than nonmelanoma skin cancer, diagnosed during the follow-up period. We excluded an additional 57 case subjects and 70 control subjects who did not have sufficient baseline serum available for the biochemical studies. Also excluded were a total of 13 case subjects and 13 control subjects with extreme values for serum estrogens and estrogen metabolites. Extreme values were defined as those for which the sum of all estrogens and estrogen metabolites was either lower than the 25 th percentile of the distribution minus three times the interquartile range, or higher than the 75 th percentile of the distribution plus three times the interquartile range.

The remaining 354 case subjects and 423 control subjects represent $80 \%$ and $81 \%$, respectively, of the 440 case and 525 control subjects who were postmenopausal and not using hormones at baseline. Of the 354 breast cancer case subjects, 75 had in situ disease and two could not be histologically confirmed and were excluded from the analyses. The final analytic sample included 277 case subjects with histologically confirmed invasive breast cancer and 423 control subjects. 
Finally, we considered how these exclusions might affect the characteristics of the study subjects. Of the 1141 potential case subjects and 1141 potential control subjects identified, similar percentages (94\%) were postmenopausal at baseline (1076 and 1073, respectively) (Supplementary Table 1, available online). However, of these postmenopausal women, case subjects were more likely ( $\mathrm{n}=636$, or $59 \%$ ) than control subjects $(\mathrm{n}=548$, or $51 \%$ ) to be users of menopausal hormone therapy at baseline, a difference we anticipated given the demonstrated association between menopausal hormone therapy use and risk of breast cancer $(18,19)$. The women not using hormone therapy at baseline, who were included in the analysis, were statistically significantly different with regard to several characteristics from those using hormone therapy, who were excluded; but the differences were similar for case subjects and control subjects (Supplementary Tables 2 and 3, respectively, available online). Specifically, among both the potential case subjects and potential control subjects, those not on hormone therapy tended to be older at baseline, black, more likely to report natural menopause, less likely to have a history of benign breast disease, and more likely to have entered the study early.

By contrast, the subsequent exclusions among non-hormone users (due to insufficient baseline serum for biochemical assays, extreme values for total estrogens and estrogen metabolites, or in situ rather than invasive breast cancer, if a case subject) resulted in only one statistically significant difference between the included and excluded case and control subjects (Supplementary Tables 4 and 5, available online). Only menopausal hormone therapy use before baseline differed statistically significantly between women included and those excluded in this analysis, and only in the control subjects. Thus, the included control subjects were reasonably representative of female participants in the PLCO who were postmenopausal and not using hormone therapy at baseline, and the included case subjects were reasonably representative of the incident cases of invasive breast cancer that occurred among these women.

\section{Laboratory Assays}

Serum samples that were stored at $-80^{\circ} \mathrm{C}$ since collection were thawed at $4^{\circ} \mathrm{C}$ and used for measurement of estrogens and estrogen metabolites. Estrone and estradiol in blood remain stable for years during long-term storage at $-70^{\circ} \mathrm{C}$ or lower (20).

Stable isotope dilution LC/MS/MS was used to measure concurrently 15 serum estrogens and estrogen metabolites, including the parent estrogens (ie, estrone and estradiol); metabolites in the 2-hydroxylation pathway (ie, 2-hydroxyestrone, 2-methoxyestrone, 2-hydroxyestradiol, 2-methoxyestradiol, and 2-hydroxyestrone-3methyl ether); metabolites in the 4-hydroxylation pathway (ie, 4-hydroxyestrone, 4-methoxyestrone, and 4-methoxyestradiol); and metabolites in the 16-hydroxylation pathway (ie, 16 $16-$ hydroxyestrone, estriol, 17-epiestriol, 16-ketoestradiol, and 16-epiestriol). Details of the method for measuring serum estrogens and estrogen metabolites, including sample preparation and assay conditions, have been published previously (16). For this study, we used six stable isotopically labeled standards to account for losses of the 15 estrogens and estrogen metabolites: deuterated 2-hydroxyestradiol, 2-methoxyestradiol and estriol (C/D/N
Isotopes Inc, Pointe-Claire, QC, Canada); deuterated 16-epiestriol (Medical Isotopes Inc, Pelham, NH); and ${ }^{13} \mathrm{C}$-labeled estrone and estradiol (Cambridge Isotope Laboratories, Andover, MA).

Serum from postmenopausal women contains 15 estrogens and estrogen metabolites. All are found in conjugated forms, attached to sulfate or glucuronide moieties; five (ie, estrone, estradiol, 2-methoxyestrone, 2-methoxyestradiol, and estriol) also exist in unconjugated form. Steps for measurement of unconjugated estrogens and estrogen metabolites in serum included addition of the six stable isotopically labeled standards, extraction with dichloromethane, derivatization with dansyl chloride, and LC/MS/MS. An additional step - enzymatic hydrolysis using a preparation from Helix pomatia with $\beta$-glucuronidase and sulfatase activity (Sigma Chemical Co, St Louis, MO)—enables the sum of the unconjugated and conjugated forms of each estrogen or estrogen metabolite to be measured. Subsequently, for each estrogen or estrogen metabolite, the quantity of the conjugated form was calculated as the difference between this sum and the measure of the unconjugated form.

Briefly, to measure estrogens and estrogen metabolites, we added the stable isotopically labeled standards to $1.0-\mathrm{mL}$ serum samples, split the serum samples into two equal aliquots, and hydrolyzed one of the two aliquots with the $\beta$-glucuronidasesulfatase enzyme preparation. We then extracted, derivatized, and performed LC/MS/MS on each aliquot. The single chemical derivatization added a dansyl group (1-dimethyl-amino-naphthalene-5sulfonyl) to the phenolic hydroxyl present on all estrogens and estrogen metabolites. The bulky charged dansyl group facilitates measuring the neutral lipophilic steroids by mass spectrometry, a technique that separates and detects compounds on the basis of charge and molecular weight.

For this study, we used serum samples from six postmenopausal women selected to cover a range of circulating estrogen and estrogen metabolite concentrations as quality control samples. Four quality control samples, including two aliquots from the same subject, were randomly inserted in every batch of approximately 40 samples. Laboratory personnel were blinded to both casecontrol status and quality control samples. Total laboratory coefficients of variation were less than $5 \%$ for all individual estrogens and estrogen metabolites measured and less than $3 \%$ for estrone, estradiol, and estriol. The published limit of quantitation (ie, the lower limit of absolute concentrations at which reliable precise readings can be obtained) is $8 \mathrm{pg} / \mathrm{mL}$ serum $(26.5-29.6 \mathrm{pmol} / \mathrm{L})$ for each estrogen and estrogen metabolite (16). However, results from this study suggest that the limit of quantitation is considerably lower. The mean serum concentration of unconjugated estradiol for the six quality control samples was $15.8 \mathrm{pmol} / \mathrm{L}$. The total laboratory coefficient of variation for unconjugated estradiol in the quality control samples was less than $2 \%$ and included both within- and between-batch variation over 6 months and all steps of the analytic procedure. Moreover, the total laboratory coefficients of variation for the two least abundant estrogen metabolites4-methoxyestradiol (mean serum concentration $=2.9 \mathrm{pmol} / \mathrm{L}$ ) and 17 -epiestriol (mean serum concentration $=1.4 \mathrm{pmol} / \mathrm{L}$ ) - were $4 \%$ and $3 \%$, respectively. These low coefficients of variation indicate that estrogen and estrogen metabolite concentrations of 1-2 pmol/L are above the limit of quantitation of our assay. No 
assays of estrogens or estrogen metabolites in this study resulted in non-detectable readings.

\section{Statistical Analysis}

Except where noted, control subjects were weighted by the inverse sampling fraction to represent the study cohort, defined as PLCO participants in the screening arm of the trial, who had completed the baseline questionnaire and at least one study update, reported no history of breast cancer at baseline, provided DNA for genotyping at baseline or during follow-up, and given written informed consent (Supplementary Table 1, available online). Case subjects were given a weight of 1 because no sampling occurred.

Estrogens and estrogen metabolites were analyzed individually, in groups representing metabolic pathways, and as ratios of metabolic pathways. Individual and grouped estrogens and estrogen metabolites were log-transformed using base 1.2 because in the study cohort, serum concentrations increased by approximately $20 \%$ between the 10th and 90th percentiles for most of these measures. Because the pathway ratios increased by approximately $4 \%$ between the 10th and 90th percentiles, they were logtransformed using base 1.04 .

Pearson correlation coefficients were calculated using control subjects weighted to represent the study cohort and log-transformed continuous measures of estrogen and estrogen metabolism. To assess differences in distributions of breast cancer risk factors between case subjects and control subjects, weighted $\chi^{2}$ tests were performed.

Hazard ratios (HRs) and 95\% confidence intervals (CIs) were calculated using weighted Cox proportional hazards regression with attained age as the time scale (21). Because we chose logarithmic bases as described above, the hazard ratio associated with a unit increase in the log-transformed continuous estrogen or estrogen metabolism measure approximately represents the relative risk comparing the highest with the lowest decile of the measure. The assumption of proportional hazards was tested by assessing plots of standardized Schoenfeld residuals vs attained age; the residuals showed no unusual patterns, which suggested no major violations of this assumption.

All Cox proportional hazards models were adjusted for study design factors, each entered categorically (age at study entry [in 5-year age groups]: 55-59, 60-64, 65-69, 70-74 years; period of blood collection: January 26, 1994, to September 29, 1997 [the median date at study entry], September 30, 1997, to October 17, 2001), and accepted breast cancer risk factors, also entered categorically (age at menarche: $<12,12-13$ or missing, $\geq 14$ years; combined parity and age at birth of first child: nulliparous, $\geq 1$ live birth and age $<19$ years, 1-2 live births and age 20-29 years, $\geq 3$ live births and age 20-29 years or missing parity or missing age, $\geq 1$ live birth and age $\geq 30$ years; age at natural menopause: $<45,45-49,50-54, \geq 55$ years, missing; type of menopause: natural menopause, surgical menopause with both ovaries removed, other or missing; previous use of menopausal hormone therapy: former, never; personal history of benign breast disease: yes, no or missing; and first-degree family history of breast cancer: yes, no or missing). Cutpoints for breast cancer risk factors are those often used in other breast cancer studies or reflect categories of response on the study questionnaire. Participants with missing data for a particular variable were assigned to the most prevalent category for that variable, as described above. Models were not adjusted for race because there were very few nonwhite subjects. None of the individual covariates modified regression coefficients associated with estrogen or estrogen metabolism measures by greater than $10 \%$.

We also assessed whether associations between $\log$-transformed measures of estrogen and estrogen metabolism and the risk of breast cancer were modified by the time from blood draw to diagnosis ( $\leq 1327$ days [median] vs $>1327$ days), by the period of blood draw (January 26, 1994, to September 29, 1997, vs September 30, 1997 , to October 17, 2001), by age at study entry on 10-year groups (55-64 vs 65-74 years), or by history of menopausal hormone therapy before study entry (ever vs never). We compared risk estimates across these strata and calculated whether corresponding interaction terms were statistically significant. We also assessed whether exclusion of case subjects diagnosed within 2 years of blood collection modified the direction or magnitude of these risk estimates.

To assess whether a linear term always provided the best fit to the data, we added a quadratic term, namely, the square of the difference between the log-transformed measure for each participant and the mean of the log-transformed measure among control subjects weighted to represent the study cohort, to the linear models described above, and then tested the statistical significance of the quadratic term. For most of the estrogen and estrogen metabolism measures, the quadratic term was not statistically significant. Nevertheless, even for associations with evidence of some deviation from linearity, we chose to present the linear models to summarize the associations of the measures of estrogen and estrogen metabolism with the risk of breast cancer.

We also present forest plots of hazard ratios for quintiles of serum concentrations of estrogens, estrogen metabolites, metabolic pathway groups, and metabolic pathway ratios, based on the distributions of these measures in control subjects weighted to represent the study cohort. These analyses required no assumptions about the linearity of the relationship of the measure of interest with breast cancer. All models were adjusted for the study design factors and accepted breast cancer risk factors described previously, with the same categorizations.

For estrogens, estrogen metabolites, metabolic pathway groups, and metabolic pathway ratios, the statistical significance of trends in risk was assessed using $P$ values associated with the log-transformed continuous measures. All $P$ values were based on two-sided tests; $P$ values less than or equal to .05 were considered statistically significant. With a sample of 277 case subjects and 423 control subjects, the power to detect a trend across quintiles, if the relative risk comparing the highest to the lowest quintile is 2.0, exceeds $80 \%$.

We did not adjust for multiple comparisons. To assess the robustness of our findings for specific estrogen and estrogen metabolism measures, we used log-transformed continuous and categorical measures, both without and with adjustment for unconjugated estradiol, in our models and emphasized the findings that were consistent across the models.

Finally, we assessed the degree to which estimates of the absolute risk of breast cancer based on the serum concentration of unconjugated estradiol alone would be altered by adding an additional estrogen or estrogen metabolism measure into the prediction model. More specifically, for each individual control subject, we first calculated the hazard ratio for unconjugated estradiol alone as 


$$
\mathrm{HR} 1=\exp \left\{\hat{\beta}_{1}\left(X_{1}-\bar{X}_{1}\right)\right\}
$$

where $X_{1}$ is the $\log$ of unconjugated estradiol for that individual control subject and $\bar{X}_{1}$ is the mean of log-transformed unconjugated estradiol among control subjects. We then calculated the hazard ratio for unconjugated estradiol and one additional estrogen or estrogen metabolism measure as

$$
H R 2=\exp \left\{\hat{\beta}_{1}^{*}\left(X_{1}-\bar{X}_{1}\right)+\hat{\beta}_{2}^{*}\left(X_{2}-\bar{X}_{2}\right)\right\},
$$

where $X_{2}$ corresponds to the $\log$ of the other measure. The models used to estimate the two hazard ratios also included the study design factors and accepted breast cancer risk factors described previously, with the same categorizations.

Absolute individual risks of breast cancer can be computed based on fitted models and the assumption that over a short interval, such as 1 year, in which competing causes of death can be ignored, the absolute risk of disease is proportional to the relative risk. To calibrate absolute risks for the sample of control subjects, we first multiplied the hazard ratio by a scale factor that was chosen so that the average risk of breast cancer in control subjects equaled the invasive breast cancer incidence rate, $\mu$, for white women aged 60-64 years in the Surveillance, Epidemiology, and End Results (SEER) population in 2004-2006 (357 breast cancers per $10^{5}$ person-years) (22). The incidence rate for each control subject corresponding to her individual estrogen and estrogen metabolite profile was calculated as $\mathrm{I} 1=(\mu \times \mathrm{HR} 1) /$ mean $(\mathrm{HR} 1)$ and $\mathrm{I} 2=(\mu \times$ HR2 $) /$ mean $(\mathrm{HR} 2)$, where mean (HR1) and mean (HR2) are the mean hazard ratios for the control subjects. The incidence rates were plotted for each control subject, with I2 on the ordinate and I1 on the abscissa. If the two rates are equal, they will fall on a diagonal line. Points outside the dotted lines on the plots indicate that the estimated rate I 2 differs from I1 by more than 50 cases per $10^{5}$ person-years.

\section{Results}

The median time from PLCO entry to breast cancer diagnosis for the case subjects was 4.3 years (interdecile range $=1.7-8.4$ years). Median follow-up among the participants in the study cohort was 8.4 years (interdecile range $=5.7-10.7$ years). The median age at entry into the study cohort was 63 years; $88 \%$ of the study cohort was non-Hispanic white. Additional characteristics of the case and control subjects in this study, and the study cohort from which they were drawn, are presented in Table 1.

The median serum concentrations of estrogens, estrogen metabolites, and metabolic pathway groups, with interdeciles ranges, for case subjects and control subjects are presented in Table 2. Only five of the 15 estrogens and estrogen metabolitesestrone, estradiol, 2-methoxyestrone, 2-methoxyestradiol, and estriol—were present in both conjugated and unconjugated forms. Among the control subjects, the parent estrogens (conjugated estrone, unconjugated estrone, conjugated estradiol, and unconjugated estradiol) constituted approximately $39 \%$ of total estrogens and estrogen metabolites $(31 \%, 5 \%, 1.8 \%$, and $1.4 \%$, respectively). Estrogen metabolites in the 2-, 4-, and 16-hydroxylation pathways accounted for approximately $12 \%, 3 \%$, and $47 \%$, respectively, of total estrogens and estrogen metabolites. Case subjects had higher median serum concentrations of nearly all estrogens, estrogen metabolites, and metabolic pathway groups compared with control subjects.

Log-transformed serum concentrations of estrogens and estrogen metabolite were moderately to highly correlated (Supplementary Table 6, available online). For example, the Pearson correlation coefficients between the serum concentration of unconjugated estradiol and serum concentrations of total estrogens and estrogen metabolites, parent estrogens, and the 2-, $4-$, and 16-hydroxylation pathways were $0.66,0.66,0.65,0.58$, and 0.66 , respectively.

For total estrogens and estrogen metabolites, parent estrogens, estrone, estradiol, and the 2-, 4-, and 16-hydroxylation pathways, including many of the metabolites in these pathways, the risk of breast cancer was increased by approximately $70 \%-80 \%$ across the interdecile range of serum concentrations, in models that included only a single estrogen or estrogen metabolism measure (Table 3). All of these associations were statistically significant except that for the 2-hydroxylation pathway. Among the parent estrogens, the serum concentration of unconjugated estradiol was most strongly associated with the risk of breast cancer $(\mathrm{HR}=2.07,95 \% \mathrm{CI}=1.19$ to $\left.3.62, P_{\text {trend }}=.01\right)$. As noted in Table 3, for some estrogen and estrogen metabolism measures, an additional quadratic term was statistically significantly associated with the risk of breast cancer, which suggests a nonlinear component for these associations.

Because of the high correlations among estrogens, estrogen metabolites, and measures of estrogen metabolism (Supplementary Table 6, available online), we examined whether any of the measures predicted risk independently of the recognized association with unconjugated estradiol. No individual estrogen, estrogen metabolite, or metabolic pathway group remained statistically significantly associated with the risk of breast cancer after adjusting for unconjugated estradiol; the hazard ratio associated with unconjugated estradiol was also attenuated in many of these same models (Table 3).

The ratio of the 2-hydroxylation pathway to parent estrogens $\left(\mathrm{HR}=0.66 ; 95 \% \mathrm{CI}=0.51\right.$ to $\left.0.87, P_{\text {trend }}=.003\right)$ and the ratio of the 2-hydroxylation pathway to the 16-hydroxylation pathway $\left(\mathrm{HR}=0.62 ; 95 \% \mathrm{CI}=0.45\right.$ to $\left.0.86, P_{\text {trend }}=.005\right)$ were statistically significantly associated with a reduced risk of breast cancer, and the ratio of 4-hydroxylation pathway catechols to 4-hydroxylation pathway methylated catechols was statistically significantly associated with an increased risk of breast cancer $(\mathrm{HR}=1.34 ; 95 \%$ $\mathrm{CI}=1.04$ to $\left.1.72, P_{\text {trend }}=.02\right)($ Table 3$)$. After adjustment for unconjugated estradiol, these hazard ratios changed minimally; however, the association between the ratio of the 2-hydroxylation pathway to the 16-hydroxylation pathway and the risk of breast cancer became non-statistically significant $(\mathrm{HR}=0.69 ; 95 \%$ $\mathrm{CI}=0.47$ to $\left.1.02, P_{\text {trend }}=.07\right)$. When we included unconjugated estradiol, the ratio of the 2-hydroxylation pathway to parent estrogens, and the ratio of 4-hydroxylation pathway catechols to 4-hydroxylation pathway methylated catechols in a single regression model, only the ratio of the 2-hydroxylation pathway to parent estrogens ( $\mathrm{HR}=0.68,95 \% \mathrm{CI}=0.48$ to 0.94$)$ and the ratio of the 4-hydroxylation pathway catechols to 4-hydroxylation pathway methylated catechols $(\mathrm{HR}=1.38,95 \% \mathrm{CI}=1.08$ to 1.77 ) remained statistically significantly associated with the risk of breast 
Table 1. Characteristics of control subjects and breast cancer case subjects

\begin{tabular}{|c|c|c|c|c|c|c|}
\hline \multirow[b]{2}{*}{ Characteristic } & \multicolumn{3}{|c|}{ Control subjects } & \multicolumn{2}{|c|}{ Case subjects } & \multirow[b]{2}{*}{$P \dagger$} \\
\hline & No. & $\%$ & Weighted \%* & No. & $\%$ & \\
\hline Age at study entry, y & & & & & & .48 \\
\hline $55-59$ & 96 & 22.7 & 25.9 & 58 & 20.9 & \\
\hline $60-64$ & 136 & 32.2 & 30.6 & 93 & 33.6 & \\
\hline $65-69$ & 123 & 29.1 & 25.5 & 77 & 27.8 & \\
\hline $70-74$ & 68 & 16.1 & 18.0 & 49 & 17.7 & \\
\hline Period of blood sample collection & & & & & & .002 \\
\hline January 26, 1994, through September 29, 1997 & 248 & 58.6 & 49.8 & 172 & 62.1 & \\
\hline September 30, 1997, through October 17, 2001 & 175 & 41.4 & 50.2 & 105 & 37.9 & \\
\hline Race $\neq$ & & & & & & .69 \\
\hline White, non-Hispanic & 373 & 88.2 & 88.2 & 245 & 88.4 & \\
\hline Black, non-Hispanic & 19 & 4.5 & 4.8 & 16 & 5.8 & \\
\hline Hispanic & 8 & 1.9 & 1.8 & 3 & 1.1 & \\
\hline Asian or Pacific Islander & 20 & 4.7 & 4.5 & 12 & 4.3 & \\
\hline American Indian & 1 & 0.2 & 0.2 & 1 & 0.4 & \\
\hline Age at menarche, $y \ddagger$ & & & & & & .04 \\
\hline$<12$ & 70 & 16.5 & 16.5 & 67 & 24.2 & \\
\hline $12-13$ or missing & 231 & 54.6 & 54.6 & 144 & 52.0 & \\
\hline$\geq 14$ & 122 & 28.8 & 28.9 & 66 & 23.8 & \\
\hline Parity $\ddagger$ & & & & & & .82 \\
\hline Nulliparous & 39 & 9.2 & 9.4 & 24 & 8.7 & \\
\hline $1-2$ live births & 118 & 27.9 & 28.2 & 84 & 30.3 & \\
\hline$\geq 3$ live births or missing & 266 & 62.9 & 62.3 & 169 & 61.0 & \\
\hline Age at birth of first child, $y \ddagger$ & & & & & & .29 \\
\hline$<20$ & 66 & 15.6 & 16.2 & 35 & 12.6 & \\
\hline 20-24 or missing & 200 & 47.3 & 47.1 & 123 & 44.4 & \\
\hline $25-29$ & 82 & 19.4 & 19.2 & 62 & 22.4 & \\
\hline$\geq 30$ & 36 & 8.5 & 8.1 & 33 & 11.9 & \\
\hline Nulliparous & 39 & 9.2 & 9.4 & 24 & 8.7 & \\
\hline Type of menopause & & & & & & .58 \\
\hline Natural & 385 & 91.0 & 90.1 & 253 & 91.3 & \\
\hline Surgical (bilateral oophorectomy) & 18 & 4.3 & 4.9 & 9 & 3.2 & \\
\hline Other or missing & 20 & 4.7 & 5.1 & 15 & 5.4 & \\
\hline Age at natural menopause, $y$ & & & & & & .69 \\
\hline$<45$ & 44 & 10.4 & 10.5 & 29 & 10.5 & \\
\hline $45-49$ & 99 & 23.4 & 22.8 & 63 & 22.7 & \\
\hline $50-54$ & 171 & 40.4 & 40.6 & 115 & 41.5 & \\
\hline$\geq 55$ & 40 & 9.5 & 9.4 & 18 & 6.5 & \\
\hline Unknown or missing & 69 & 16.3 & 16.7 & 52 & 18.8 & \\
\hline Family history of breast cancer in first-degree relativesł & 76 & 18.0 & 18.4 & 50 & 18.1 & .90 \\
\hline Personal history of benign breast disease $\ddagger$ & 85 & 20.1 & 20.0 & 74 & 26.7 & .04 \\
\hline Previous use of menopausal hormone therapy & 166 & 39.2 & 39.8 & 92 & 33.2 & .08 \\
\hline
\end{tabular}

* Percentages reflect weighted counts and refer to the study cohort.

$+P$ values are based on two-sided $\chi^{2}$ tests that weight control subjects to make them representative of the study cohort.

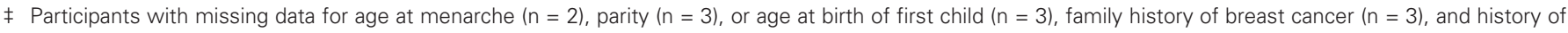
benign breast disease $(n=12)$ were assigned to the most prevalent categories for those variables. Two control subjects had missing data for race. Missing data for other variables were handled as a separate category as indicated.

cancer, whereas the association between unconjugated estradiol and the risk of breast cancer was substantially attenuated and no longer statistically significant $(\mathrm{HR}=1.31 ; 95 \% \mathrm{CI}=0.65$ to 2.64$)$.

Figure 2 shows forest plots of hazard ratios for breast cancer by quintiles of unconjugated estradiol, total estrogens, and estrogen metabolites, and the three measures that showed evidence of estradiol-independent associations with the risk of breast cancer (ie, the ratio of the 2-hydroxylation pathway to parent estrogens, the ratio of the 2-hydroxylation pathway to the 16-hydroxylation pathway, and the ratio of 4-hydroxylation pathway catechols to 4-hydroxylation pathway methylated catechols). Plots for total estrogens and estrogen metabolites and the three measures of estrogen metabolism are presented without and with adjustment for unconjugated estradiol. In general, patterns of association were neither strictly monotonic nor indicative of a threshold effect; however, the $95 \%$ confidence intervals around the hazard ratio for each quintile were wide. Forest plots of hazard ratios for breast cancer by quintiles of the other individual estrogens and estrogen metabolites, the metabolic pathway groups, and the other metabolic pathway ratios are given in Supplementary Figure 1 (available online).

We assessed whether the risk associations for all estrogen and estrogen metabolism measures listed in Figure 2 were modified by time from blood draw to diagnosis, by period of blood draw (and 
Table 2. Serum concentrations of estrogens and estrogen metabolites (in pmol/L) in conjugated* and unconjugated forms, measured in 423 control subjects weighted to represent the study cohort and 277 case subjects with incident invasive breast cancer

\begin{tabular}{|c|c|c|}
\hline & Weighted control subjects & Case subjects \\
\hline Estrogen and estrogen metabolism measures & Median (10th-90th percentiles) & Median (10th-90th percentiles) \\
\hline Total estrogens and estrogen metabolites & $1099(1025-1222)$ & $1115(1030-1228)$ \\
\hline Parent estrogens & $425.8(398.3-481.7)$ & $432.9(399.1-482.2)$ \\
\hline Estrone & $390.0(364.9-444.0)$ & $396.7(365.5-444.2)$ \\
\hline Conjugated estrone & $335.8(313.1-384.7)$ & $341.7(313.1-385.2)$ \\
\hline Unconjugated estrone & $53.95(50.54-59.43)$ & $54.46(50.54-59.57)$ \\
\hline Estradiol & $36.00(32.64-37.99)$ & $36.65(32.76-38.06)$ \\
\hline Conjugated estradiol & $20.28(17.75-22.00)$ & $20.71(17.78-21.97)$ \\
\hline Unconjugated estradiol & $15.45(14.54-16.77)$ & $15.68(14.59-16.83)$ \\
\hline 2-Hydroxylation pathway & $128.1(120.1-137.9)$ & $129.3(120.3-139.5)$ \\
\hline 2-Hydroxylation pathway catechols & $104.7(97.81-112.7)$ & $105.6(98.24-114.3)$ \\
\hline 2-Hydroxyestrone & $68.82(64.18-74.30)$ & $69.45(64.50-75.27)$ \\
\hline 2-Hydroxyestradiol & $35.86(33.60-38.78)$ & $36.06(33.55-39.05)$ \\
\hline 2-Hydroxylation pathway methylated catechols & $23.45(22.00-25.11)$ & $23.60(22.07-25.37)$ \\
\hline 2-Methoxyestrone & $12.42(11.63-13.35)$ & $12.52(11.61-13.40)$ \\
\hline Conjugated 2-methoxyestrone & $5.00(4.36-5.64)$ & $5.01(4.27-5.70)$ \\
\hline Unconjugated 2-methoxyestrone & $7.45(7.03-7.95)$ & $7.49(7.09-8.00)$ \\
\hline 2-Methoxyestradiol & $7.63(7.10-8.14)$ & $7.71(7.13-8.23)$ \\
\hline Conjugated 2-methoxyestradiol & $3.10(2.68-3.46)$ & $3.18(2.66-3.52)$ \\
\hline Unconjugated 2-methoxyestradiol & $4.56(4.22-4.85)$ & $4.57(4.24-4.86)$ \\
\hline 2-Hydroxyestrone-3-methyl ether & $3.42(3.22-3.72)$ & $3.45(3.22-3.77)$ \\
\hline 4-Hydroxylation pathway & $28.23(26.57-30.71)$ & $28.48(26.58-31.11)$ \\
\hline 4-Hydroxylation pathway catechols & + & + \\
\hline 4-Hydroxyestrone & $22.01(20.64-23.97)$ & $22.27(20.72-24.25)$ \\
\hline 4-Hydroxylation pathway methylated catechols & $6.23(5.81-6.76)$ & $6.23(5.82-6.89)$ \\
\hline 4-Methoxyestrone & $3.45(3.22-3.72)$ & $3.46(3.21-3.77)$ \\
\hline 4-Methoxyestradiol & $2.78(2.58-3.07)$ & $2.80(2.59-3.12)$ \\
\hline 16-Hydroxylation pathway & $516.6(480.5-573.5)$ & $524.3(483.1-576.2)$ \\
\hline $16 \alpha$-Hydroxyestrone & $39.03(36.12-42.50)$ & $39.44(36.11-42.72)$ \\
\hline Estriol & $436.9(404.0-486.9)$ & 443.1 (406.9-488.4) \\
\hline Conjugated estriol & $390.7(359.4-437.0)$ & $396.3(361.8-438.6)$ \\
\hline Unconjugated estriol & $46.83(43.07-50.85)$ & $47.51(43.14-51.00)$ \\
\hline 17-Epiestriol & $1.34(1.25-1.46)$ & $1.35(1.25-1.47)$ \\
\hline 16-Ketoestradiol & $36.30(34.32-39.74)$ & $36.82(34.42-40.10)$ \\
\hline 16-Epiestriol & $3.49(3.23-3.78)$ & $3.53(3.25-3.83)$ \\
\hline
\end{tabular}

therefore by the length of time blood was stored before assay), by age at study entry, or by history of menopausal hormone therapy; no statistically significant interactions were noted (data not shown). Similarly, exclusion of case subjects who were diagnosed with breast cancer within 2 years of blood collection did not modify the risk estimates in a meaningful way (data not shown).

Next, we assessed the degree to which information about total estrogens and estrogen metabolites or the three metabolic pathway ratios of interest would alter estimates of the absolute risk of breast cancer that were based on the serum concentration of unconjugated estradiol alone in a hypothetical population of women with estrogen and estrogen metabolite profiles similar to those observed in our cohort. We plotted the breast cancer rate estimate associated with the estrogen and estrogen metabolite profile of each control subject, which was calculated from models that included both unconjugated estradiol and an additional measure of interest, against the breast cancer rate estimate for each control subject derived from models that included only unconjugated estradiol (Figure 3). The rate estimates were calibrated to the 2004-2006
SEER incidence rate for white women aged 60-64 years, which was 357 breast cancers per $10^{5}$ person-years. When total estrogens and estrogen metabolites were included in the model with unconjugated estradiol, less than $14 \%$ of women had a breast cancer rate estimate that changed by more than 50 cancers per $10^{5}$ personyears (Figure 3, A). By contrast, breast cancer rate estimates changed by greater than 50 cancers per $10^{5}$ person-years for $36 \%$, $30 \%$, and $23 \%$ of women when the ratio of the 2 -hydroxylation pathway to parent estrogens (Figure 3, B), the ratio of the 2-hydroxylation pathway to the 16-hydroxylation pathway (Figure 3, C), and the ratio of 4-hydroxylation pathway catechols to 4-hydroxylation pathway methylated catechols (Figure 3, D), respectively, were added to a model with unconjugated estradiol.

\section{Discussion}

In this study, serum concentrations of unconjugated estradiol, unconjugated estrone, conjugated estrone, and many estrogen metabolites were statistically significantly associated with an 
Table 3. Hazard ratios (HRs) and 95\% confidence intervals (Cls) for invasive breast cancer associated with the change in risk across the interdecile range of serum concentrations of estrogens and estrogen metabolites, individually, in metabolic pathway groups, and as metabolic pathway ratios*

\begin{tabular}{|c|c|c|c|}
\hline Estrogen and estrogen metabolism measures & $\begin{array}{c}\text { HR }(95 \% \mathrm{Cl}) \text { for } \\
\text { estrogen or estrogen } \\
\text { metabolism measuret }\end{array}$ & $\begin{array}{c}\text { HR }(95 \% \mathrm{Cl}) \text { for } \\
\text { estrogen or estrogen } \\
\text { metabolism measureł }\end{array}$ & $\begin{array}{l}\text { HR }(95 \% \mathrm{CI}) \text { for } \\
\text { unconjugated } \\
\text { estradiolf }\end{array}$ \\
\hline Total estrogens and estrogen metabolites & 1.76 (1.09 to 2.85$)$ & 1.35 (0.69 to 2.61$)$ & $1.65(0.77$ to 3.55$)$ \\
\hline Parent estrogens§ & $1.73(1.11$ to 2.71$)$ & $1.38(0.75$ to 2.54$)$ & $1.59(0.74$ to 3.42$)$ \\
\hline Estrone§ & $1.71(1.10$ to 2.63$)$ & $1.37(0.76$ to 2.46$)$ & $1.59(0.75$ to 3.40$)$ \\
\hline Conjugated estrone§ & 1.62 (1.08 to 2.43$)$ & 1.32 (0.79 to 2.23$)$ & 1.64 (0.81 to 3.36$)$ \\
\hline Unconjugated estrone & $1.77(1.07$ to 2.95$)$ & $1.02(0.36$ to 2.92$)$ & $2.03(0.64$ to 6.42$)$ \\
\hline Estradiol & 1.75 (1.03 to 2.98$)$ & 1.21 (0.59 to 2.52$)$ & $1.81(0.85$ to 3.90$)$ \\
\hline Conjugated estradiol & $1.28(0.88$ to 1.86$)$ & $1.12(0.74$ to 1.69$)$ & 1.97 (1.09 to 3.57$)$ \\
\hline Unconjugated estradiol & 2.07 (1.19 to 3.62$)$ & NA & NA \\
\hline 2-Hydroxylation pathway & $1.73(0.93$ to 3.22$)$ & $1.08(0.47$ to 2.53$)$ & $1.98(0.93$ to 4.21$)$ \\
\hline 2-Hydroxylation pathway catechols§ & $1.72(0.94$ to 3.16$)$ & $1.10(0.48$ to 2.53$)$ & $1.96(0.91$ to 4.20$)$ \\
\hline 2-Hydroxyestrone & $1.92(1.08$ to 3.42$)$ & $1.37(0.62$ to 3.02$)$ & $1.69(0.78$ to 3.63$)$ \\
\hline 2-Hydroxyestradiol§ & 1.16 (0.57 to 2.39$)$ & $0.70(0.30$ to 1.63$)$ & $2.51(1.22$ to 5.18$)$ \\
\hline 2-Hydroxylation pathway methylated catechols & 1.56 (0.83 to 2.93$)$ & $0.99(0.45$ to 2.16$)$ & 2.09 (1.05 to 4.15$)$ \\
\hline 2-Methoxyestrone & $1.34(0.75$ to 2.42$)$ & $0.88(0.44$ to 1.78$)$ & $2.21(1.14$ to 4.26$)$ \\
\hline Conjugated 2-methoxyestrone & $0.96(0.71$ to 1.29$)$ & $0.91(0.67$ to 1.22$)$ & $2.13(1.21$ to 3.75$)$ \\
\hline Unconjugated 2-methoxyestrone & 2.14 (1.11 to 4.14$)$ & 1.33 (0.51 to 3.45$)$ & 1.74 (0.78 to 3.89$)$ \\
\hline 2-Methoxyestradiol & $1.66(0.91$ to 3.03$)$ & $1.13(0.55$ to 2.33$)$ & 1.95 (0.99 to 3.84$)$ \\
\hline Conjugated 2-methoxyestradiol & $1.17(0.87$ to 1.60$)$ & $1.11(0.81$ to 1.51$)$ & 2.01 (1.14 to 3.55$)$ \\
\hline Unconjugated 2-methoxyestradiol & 1.54 (0.84 to 2.83$)$ & $0.76(0.32$ to 1.78$)$ & $2.50(1.13$ to 5.53$)$ \\
\hline 2-Hydroxyestrone-3-methyl ether & $1.60(0.92$ to 2.80$)$ & $1.14(0.58$ to 2.25$)$ & $1.93(0.98$ to 3.80$)$ \\
\hline 4-Hydroxylation pathway & $1.81(1.03$ to 3.18$)$ & $1.31(0.64$ to 2.66$)$ & $1.78(0.88$ to 3.60$)$ \\
\hline 4-Hydroxylation pathway catechols & $\|$ & $\|$ & $\|$ \\
\hline 4-Hydroxyestrone & $1.91(1.10$ to 3.31$)$ & $1.43(0.72$ to 2.85$)$ & $1.68(0.84$ to 3.39$)$ \\
\hline 4-Hydroxylation pathway methylated catechols & 1.34 (0.76 to 2.35$)$ & $0.88(0.43$ to 1.77$)$ & $2.24(1.11$ to 4.49$)$ \\
\hline 4-Methoxyestrone & 1.19 (0.67 to 2.12$)$ & $0.74(0.36$ to 1.52$)$ & 2.45 (1.22 to 4.92$)$ \\
\hline 4-Methoxyestradiol & $1.40(0.87$ to 2.26$)$ & $1.05(0.60$ to 1.84$)$ & $2.01(1.04$ to 3.89$)$ \\
\hline 16-Hydroxylation pathway & 1.74 (1.08 to 2.80$)$ & 1.33 (0.69 to 2.55$)$ & 1.66 (0.77 to 3.56$)$ \\
\hline $16 \alpha$-Hydroxyestrone & 1.51 (0.91 to 2.49$)$ & $1.06(0.56$ to 2.02$)$ & $2.00(0.98$ to 4.06$)$ \\
\hline Estriol & 1.69 (1.07 to 2.66$)$ & 1.31 (0.71 to 2.42$)$ & $1.67(0.78$ to 3.56$)$ \\
\hline Conjugated estriol§ & $1.63(1.05$ to 2.51$)$ & $1.28(0.73$ to 2.24$)$ & $1.71(0.83$ to 3.51$)$ \\
\hline Unconjugated estriol & $1.76(1.06$ to 2.92$)$ & $1.02(0.36$ to 2.89$)$ & $2.04(0.64$ to 6.51$)$ \\
\hline 17-Epiestriol & 1.43 (0.84 to 2.44$)$ & $0.99(0.51$ to 1.91$)$ & 2.09 (1.05 to 4.16$)$ \\
\hline 16-Ketoestradiol & $1.98(1.14$ to 3.44$)$ & $1.54(0.79$ to 3.02$)$ & $1.63(0.83$ to 3.22$)$ \\
\hline 16-Epiestriol & $1.83(1.08$ to 3.11$)$ & $1.40(0.76$ to 2.59$)$ & $1.71(0.90$ to 3.27$)$ \\
\hline \multicolumn{4}{|l|}{ Metabolic pathway ratios } \\
\hline 2-Hydroxylation pathway:parent estrogens§ & 0.66 (0.51 to 0.87$)$ & $0.72(0.52$ to 1.00$)$ & $1.45(0.73$ to 2.85$)$ \\
\hline 4-Hydroxylation pathway:parent estrogens & $0.87(0.73$ to 1.04$)$ & $0.94(0.77$ to 1.14$)$ & $1.92(1.05$ to 3.51$)$ \\
\hline 16-Hydroxylation pathway:parent estrogens & $0.52(0.26$ to 1.06$)$ & $0.64(0.32$ to 1.31$)$ & 1.86 (1.04 to 3.33$)$ \\
\hline 2-Hydroxylation pathway:16-hydroxylation pathway§ & $0.62(0.45$ to 0.86$)$ & $0.69(0.47$ to 1.02$)$ & $1.53(0.79$ to 2.96$)$ \\
\hline 2-Hydroxylation pathway:4-hydroxylation pathway & $0.86(0.64$ to 1.17$)$ & $0.85(0.63$ to 1.14$)$ & $2.12(1.21$ to 3.70$)$ \\
\hline 4-Hydroxylation pathway:16-hydroxylation pathway & $0.90(0.75$ to 1.08$)$ & $0.97(0.80$ to 1.18$)$ & $2.02(1.12$ to 3.62$)$ \\
\hline 2-Hydroxylation pathway catechols:methylated catechols & 1.15 (0.87 to 1.53$)$ & $1.06(0.79$ to 1.42$)$ & $2.01(1.13$ to 3.57$)$ \\
\hline 4-Hydroxylation pathway catechols:methylated catechols & $1.34(1.04$ to 1.72$)$ & $1.31(1.03$ to 1.68$)$ & $2.03(1.15$ to 3.56$)$ \\
\hline
\end{tabular}

* All models were adjusted for age at study entry: 55-59, 60-64, 65-69, 70-74 years; period of blood collection: January 26, 1994, to September 29, 1997, September 30, 1997, to October 17, 2001; age at menarche: $<12,12-13$ or missing, $\geq 14$ years; combined parity and age at birth of first child: nulliparous, $\geq 1$ live births and age $<20$ years, 1-2 live births and age 20-29 years, $\geq 3$ live births and age 20-29 years or missing parity or missing age, $\geq 1$ live births and age $\geq 30$ years; age at natural menopause: $<45,45-49,50-54, \geq 55$ years, missing; type of menopause: natural menopause, surgical menopause with both ovaries removed, other or missing; first-degree family history of breast cancer: yes, no or missing; personal history of benign breast disease: yes, no or missing; previous

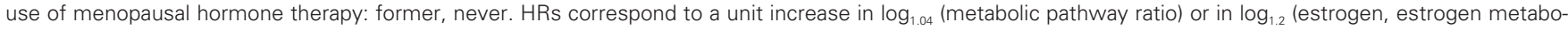
lite, or metabolic pathway group). These logarithmic bases were chosen so that a unit increase in the logarithm corresponds approximately to an increase in the measure from the 10th to the 90th percentile (in weighted control subjects). NA = not applicable.

† Model includes the estrogen or estrogen metabolism measure in addition to the covariates listed above.

₹ Model includes unconjugated estradiol, the estrogen or estrogen metabolism measure, and the covariates listed above.

$\S$ Risk associations for these measures were found to have a statistically significant quadratic component in addition to the linear component. Although these associations show deviation from linearity, the linear relation is presented here as a convenient summary description.

|| 4-Hydroxyestrone is the only 4-hydroxylation pathway catechol. Therefore, estimates of association are the same for both these measures. 
increased risk of postmenopausal breast cancer. The risk of breast cancer was doubled when women in the highest decile of serum concentration were compared with women in the lowest decile, a finding that is consistent with a pooled analysis of prospective data available worldwide (1). The sum of all the estrogens and estrogen metabolites measured, including conjugated and unconjugated forms, was not more strongly associated with the risk of breast cancer compared with unconjugated estradiol.

We identified three metabolic pathway ratios that were associated with the risk of breast cancer even after adjustment for circulating levels of unconjugated estradiol: the ratio of the 2-hydroxylation pathway to parent estrogens, the ratio of the 2-hydroxylation pathway to the 16-hydroxylation pathway, and the ratio of 4-hydroxylation pathway catechols to 4-hydroxylation pathway methylated catechols. We found that inclusion of any of these three measures of estrogen metabolism in a model appreciably changed the estimated absolute risk of breast cancer for many women compared with risk predicted on the basis of unconjugated estradiol alone.

The ratio of the 2-hydroxylation pathway to parent estrogens was associated with a statistically significantly decreased risk of breast cancer after adjustment for unconjugated estradiol. In this study, this ratio was more strongly associated with the risk of breast cancer compared with the ratio of 2-hydroxylation pathway to 16-hydroxylation pathway or unconjugated estradiol alone. Compared with estrogens and other estrogen metabolites, 2-hydroxylation pathway catechols have relatively low affinities for estrogen receptors (4) and are rapidly cleared from circulation (23). Thus, 2-hydroxylation may result in a decrease in bioavailable estrogens and reduced estrogen receptor-mediated signaling in the breast.

In this study, the ratio of the 2-hydroxylation pathway to the 16-hydroxylation pathway was associated with a non-statistically significantly decreased risk of breast cancer after adjustment for unconjugated estradiol ( $\mathrm{HR}=0.69 ; 95 \% \mathrm{CI}=0.47$ to 1.02$)$. Although this study is the first to our knowledge to evaluate the entire 2- and 16-hydroxylation pathways in postmenopausal women, a number of epidemiological studies have assessed the risk of postmenopausal breast cancer in association with concentrations of 2-hydroxyestrone, 16 $\alpha$-hydroxyestrone, or their ratio, as measured in urine or blood. Two early retrospective studies with 42 (24) and 65 (25) breast cancer case subjects each found a statistically significant, inverse association between the ratio of 2-hydroxyestrone to $16 \alpha$-hydroxyestrone in urine and the risk of breast cancer. Other epidemiological studies of these metabolites, including some larger retrospective studies (26-28) and all prospective studies conducted to date (29-35), have not provided clear support for the hypothesis that this ratio is associated with reduced breast cancer risk. Although there were some statistically significant subgroup findings in some of the prospective studies $(27,33,34)$, they were not consistently reported across studies. All previous studies relied on immunoassays to measure estrogens and estrogen metabolites, and these mixed largely negative findings may reflect limitations in the specificity, sensitivity, and reproducibility of those assays (36).

To our knowledge, this is the first epidemiological study of breast cancer risk with direct measures of circulating estrogen metabolites in the 4-hydroxylation pathway. In this study, the ratio of catechols to methylated catechols in the 4-hydroxylation pathway was associated with statistically significantly increased risk of breast cancer. This result is consistent with the hypothesis that mutagenic quinones derived from 4-hydroxylation pathway catechols contribute to pathogenesis of postmenopausal breast cancer. Catechols in both the 2- and 4-hydroxylation pathways can be oxidized to form quinones; these reactive electrophiles can then react with DNA to form a variety of adducts $(37,38)$. Methylation of the catechols prevents their conversion to reactive quinones (39). Whereas the most common DNA adducts derived from 4-hydroxylation pathway catechols are depurinating and highly mutagenic $(7,40)$, most of those derived from 2-hydroxylation pathway catechols are stable and can be repaired with little error (5); this difference may explain why 2-hydroxylation pathway catechols are not potent carcinogens in animal models of estrogen-mediated cancers (41-43) and why their ratio to the corresponding methylated catechols was not statistically significantly associated with the risk of breast cancer in this study.

This study has several notable strengths. The PLCO cohort is a large prospective study with standardized specimen collection and storage. The estrogen and estrogen metabolite profiles resulting from the LC/MS/MS assay included five estrogens and estrogen metabolites found in circulation in both conjugated and unconjugated forms and 10 found in conjugated form only, and therefore provide a novel phenotypic characterization of individual patterns of estrogen metabolism. Most previous epidemiological tests of hypotheses about associations between estrogen metabolism and breast cancer relied on immunoassays, which were available only for a few estrogens and estrogen metabolites and had limited sensitivity, specificity, and reproducibility, especially at the low serum and urinary concentrations that are typical of postmenopausal women $(14,15)$. Other tests of these hypotheses have been based on measurements of common genetic polymorphisms in estrogen metabolism pathways, which are likely to have small individual effects on the estrogen and estrogen metabolite phenotype $(44,45)$.

This study also has four major limitations. First, consistent with most previous studies of endogenous steroid hormone levels and risk of postmenopausal breast cancer, we included only case subjects and control subjects who were postmenopausal and not using menopausal hormone therapy at the time of blood collection. This decision may limit the generalizability of the findings but allowed us to investigate endogenous estrogen metabolism unperturbed by exogenous estrogens. However, it is reassuring to note that associations between estrogen and estrogen metabolism measures and the risk of breast cancer did not differ between subgroups of women who had and had not used menopausal hormone therapy before PLCO study entry.

A second concern is the limited interindividual variation in serum concentrations of estrogens and estrogen metabolites observed in this study. There was less variability in serum concentrations of estradiol and estrone in our study sample compared with those reported in previous studies of postmenopausal women that relied on immunoassays. The lower concentrations and more limited ranges observed in this study are consistent with the improved specificity of our assay, as shown in a recent comparison of urinary estrogen and estrogen metabolite measures obtained using traditional immunoassays and the LC/MS/MS technique 

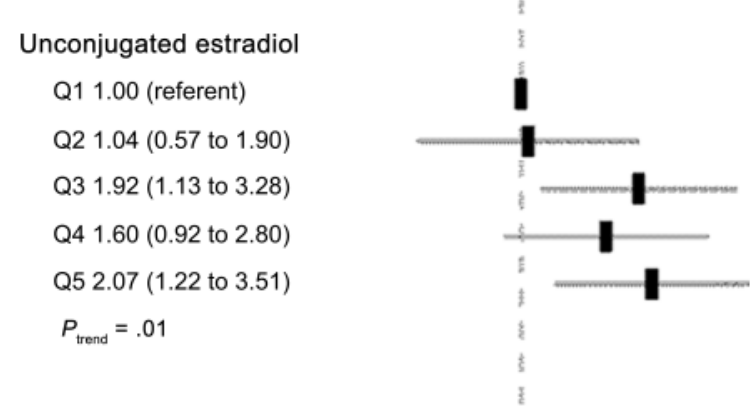

Total estrogens and estrogen metabolites

Q1 1.00 (referent)

Q2 0.90 (0.53 to 1.56 )

Q3 1.10 (0.63 to 1.90$)$

Q4 0.86 (0.50 to 1.50$)$

Q5 1.70 (1.03 to 2.80 )

$P_{\text {trend }}=.02$

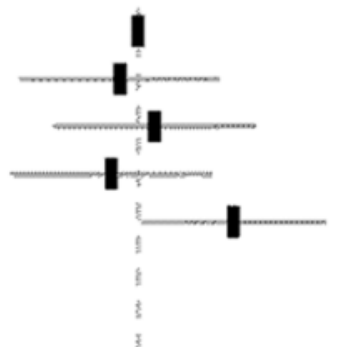

2-Hydroxylation pathway : parent estrogens

Q1 1.00 (referent)

Q2 0.66 (0.40 to 1.11)

Q3 0.53 (0.31 to 0.90 )

Q4 0.67 (0.40 to 1.14$)$

Q5 0.54 (0.32 to 0.90$)$

$P_{\text {trend }}=.003$

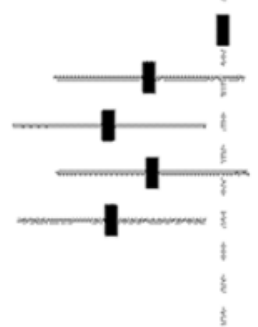

2-Hydroxylation pathway : 16-hydroxylation pathway

Q1 1.00 (referent)

Q2 0.91 (0.55 to 1.51)

Q3 0.62 (0.35 to 1.08$)$

Q4 0.53 (0.31 to 0.91$)$

Q5 0.64 (0.38 to 1.07)

$P_{\text {trond }}=.005$

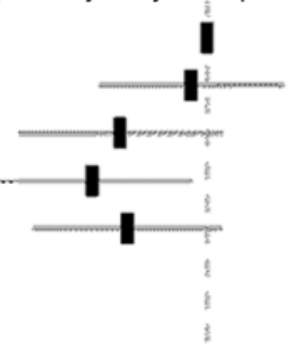

4-Hydroxylation pathway catechols : methylated catechols Q1 1.00 (referent)

Q2 1.50 (0.89 to 2.51)

Q3 1.19 (0.69 to 2.05)

Q4 0.92 (0.53 to 1.58$)$

Q5 1.76 (1.06 to 2.93)

$P_{\text {trend }}=.02$

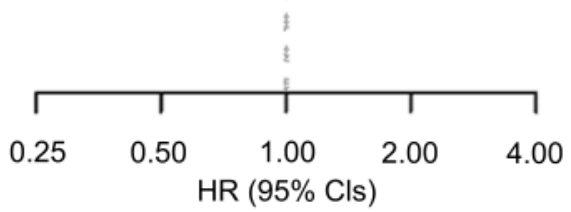

Figure 2. Forest plots of hazard ratios (HRs) for invasive breast cancer and $95 \%$ confidence intervals (Cls) by quintiles of unconjugated estradiol, total estrogens and estrogen metabolites, and selected metabolic pathway ratios. HRs (black rectangles) are shown on a log scale. Gray lines represent $95 \%$ Cls. Quintiles are abbreviated Q1, Q2, Q3, Q4, and Q5 and were defined based on distributions of these measures among
Total estrogens and estrogen metabolites

Q1 1.00 (referent)

Q2 0.85 (0.49 to 1.46$)$

Q3 0.92 (0.50 to 1.69)

Q4 0.68 (0.35 to 1.31$)$

Q5 1.24 (0.64 to 2.38$)$

$P_{\text {trend }}=.38$

2-Hydroxylation pathway : parent estrogens

Q1 1.00 (referent)

Q2 0.69 (0.41 to 1.16$)$

Q3 0.63 (0.36 to 1.12$)$

Q4 0.86 (0.46 to 1.58$)$

Q5 0.67 (0.37 to 1.20$)$

$P_{\text {trond }}=.05$

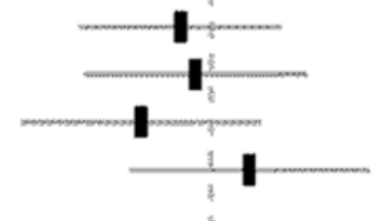

2-Hydroxylation pathway : 16-hydroxylation pathway

Q1 1.00 (referent)

Q2 0.92 (0.55 to 1.51)

Q3 0.68 (0.38 to 1.22$)$

Q4 0.62 (0.34 to 1.14$)$

Q5 0.74 (0.41 to 1.34$)$

$P_{\text {trend }}=.07$
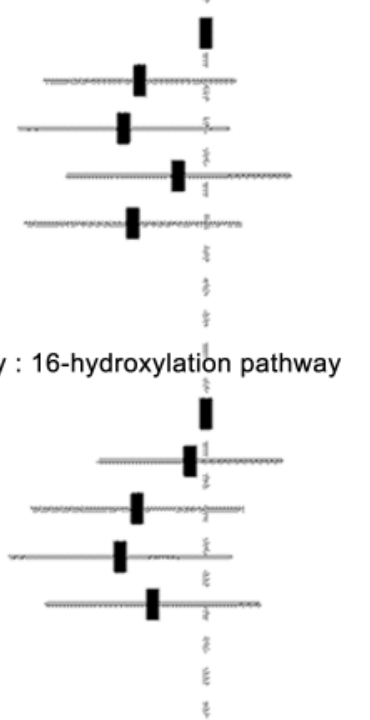

4-Hydroxylation pathway catechols : methylated catechols

Q1 1.00 (referent)

Q2 1.51 (0.90 to 2.54)

Q3 1.33 (0.76 to 2.33 )

Q4 1.07 (0.61 to 1.89$)$

Q5 1.81 (1.09 to 3.03)

$P_{\text {trend }}=.03$

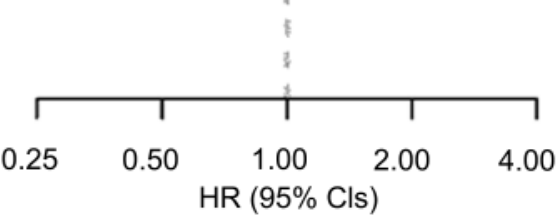

control subjects weighted to represent the study cohort. Models shown on the right-hand side were adjusted for log-transformed (to the base 1.2) continuous unconjugated estradiol. All models were adjusted for age at study entry: 55-59, 60-64, 65-69, 70-74 years; period of blood collection: January 26, 1994, to September 29, 1997, September 30, 1997, to October 17, 2001; age at menarche: $<12,12-13$ or missing, $\geq 14$ years; 

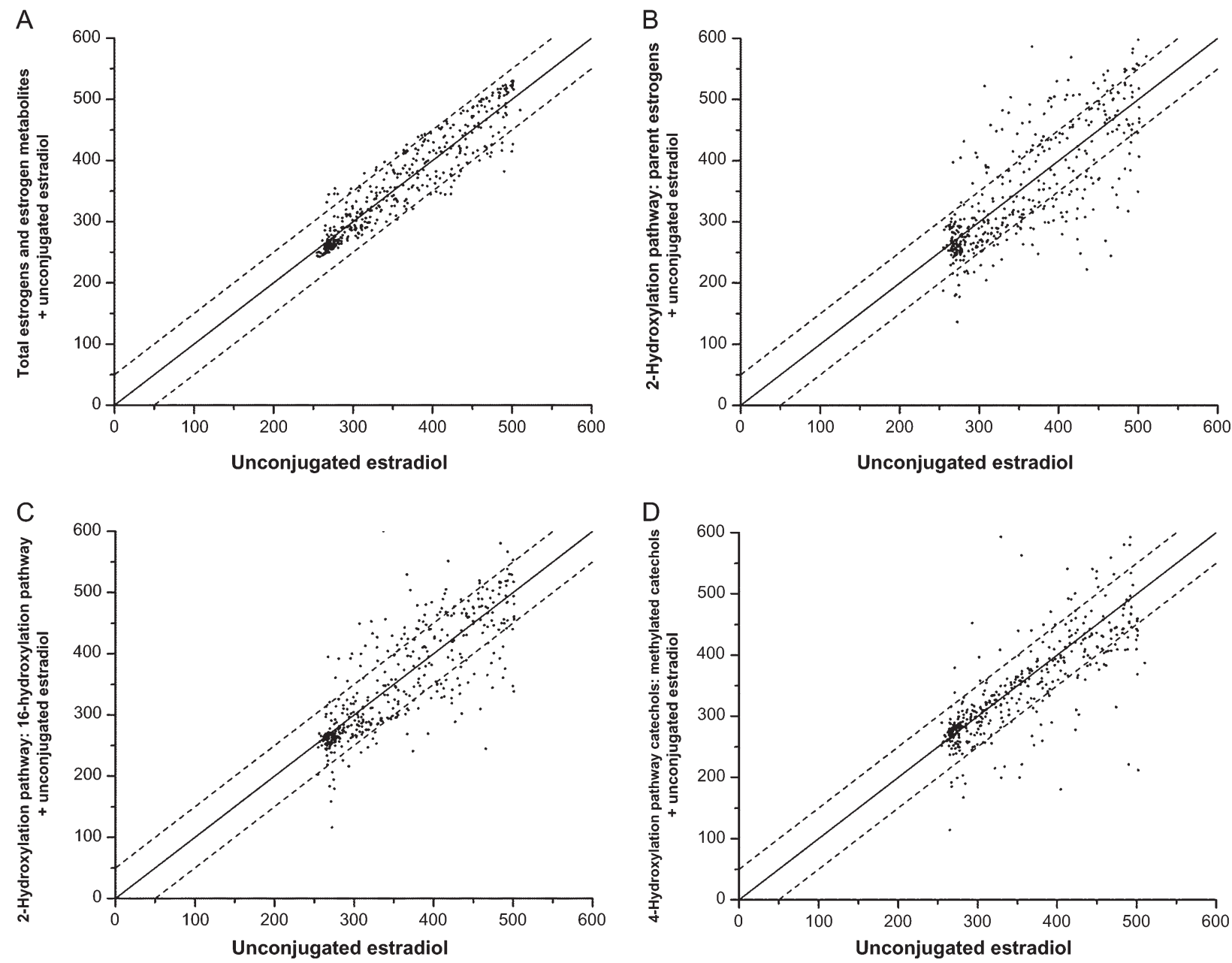

Figure 3. Comparison of estimated absolute risks of breast cancer (expressed as incidence rates, in cases per $10^{5}$ person-years) for each study control subject. Estimated risks were based on relative hazard (Cox) models for the estrogen and estrogen metabolite profile of each control subject. One model included unconjugated estradiol only $(x$-axis), and the other model included both unconjugated estradiol and an additional measure of interest ( $y$-axis): total estrogens and estrogen metabolites (A); the ratio of the 2-hydroxylation pathway to parent estrogens (B); the ratio of the 2-hydroxylation pathway to the 16-hydroxylation pathway (C); and the ratio of 4-hydroxylation pathway catechols to 4-hydroxylation pathway methylated catechols (D). Absolute risk estimates were calibrated using breast cancer incidence rates for white women, aged 60-64 years, in the 2004-2006 Surveillance, Epidemiology, and End Results population (357 cases per $10^{5}$ personyears), as described in the "Methods" section. Each dot represents two estimated absolute risks for each control subject. If the two risks are

used in this study (15). A third limitation is that serum concentrations of most estrogens and estrogen metabolites were highly correlated in this study. Limited interindividual variability in concentrations of estrogens and estrogen metabolites and high correlations among many of these measures mean that definitive

equal, they will fall on a diagonal line. Upper and lower dashed lines demarcate risk predictions that differ by at least 50 cases per $10^{5}$ person-years. To estimate relative hazards from Cox models, estrogen and estrogen metabolism measures were log-transformed and the following covariates were included: age at study entry: 55-59, 60-64, 65$69,70-74$ years; period of blood collection: January 26,1994 , to September 29, 1997, September 30,1997, to October 17, 2001; age at menarche: $<12$, $12-13$ or missing, $\geq 14$ years; combined parity and age at birth of first child: nulliparous, $\geq 1$ live birth and age $<20$ years, $1-2$ live births and age 20-29 years, $\geq 3$ live births and age 20-29 years or missing parity or missing age, $\geq 1$ live birth and age $\geq 30$ years; age at natural menopause: $<45,45-49$, $50-54, \geq 55$ years, missing; type of menopause: natural menopause, surgical menopause with both ovaries removed, other or missing; firstdegree family history of breast cancer: yes, no or missing; personal history of benign breast disease: yes, no or missing; previous use of menopausal hormone therapy: former, never.

identification of any individual estrogens, or estrogen metabolites with independent roles in carcinogenesis may require very large sample sizes.

A fourth concern arises from the number of comparisons made in the present analysis. Because our LC/MS/MS method permitted

\section{Figure 2 (continued).}

combined parity and age at birth of first child: nulliparous, $\geq 1$ live birth and age $<20$ years, $1-2$ live births and age 20-29 years, $\geq 3$ live births and age $20-29$ years or missing parity or missing age, $\geq 1$ live birth and age $\geq 30$ years; age at natural menopause: $<45,45-49,50-54, \geq 55$ years, missing; type of menopause: natural menopause, surgical menopause with both ovaries removed, other or missing; first-degree family history of breast cancer: yes, no or missing; personal history of benign breast disease: yes, no or missing; previous use of menopausal hormone therapy: former, never. $P$ for trend was based on two-sided Wald tests of the coefficient associated with a unit increase in $\log _{12}$-transformed unconjugated estradiol, in $\log _{1.2}$-transformed total estrogens and estrogen metabolites, or in $\log _{1.04}$-transformed metabolic pathway ratios. 
study of circulating levels of 15 individual estrogens and estrogen metabolites for the first time in an epidemiological study, we systematically examined risk associations for each of them: 10 were detected only in conjugated forms and five in both conjugated and unconjugated forms. We also evaluated the associations with a number of derived measures, including the sum of all estrogens and estrogen metabolites, seven metabolic pathway groups, and eight metabolic pathway ratios. These groups and ratios were motivated by recognized metabolic pathways, shared biochemistry, and etiologic hypotheses. We have not adjusted for multiple comparisons because our primary aim was to identify promising predictive measures. Thus, it is possible that some of our findings are due to chance. We have some confidence in our findings because the associations with unconjugated and conjugated estrone and unconjugated estradiol that we report have been previously established (1). We recognize that all of our results require independent confirmation in additional welldesigned studies.

Much of the experimental research on estrogen-mediated carcinogenesis over the last 30 years has focused on whether the underlying mechanism is related to the role of estrogen as a mitogen that stimulates breast cell proliferation through activation of the estrogen receptor, or its role as a precursor to a potent mutagen. Our findings support the recent suggestion (13) that both of these mechanisms may contribute to estrogen-mediated carcinogenesis. Specifically, our results point to three estrogen-related factors that may contribute to breast cancer pathogenesis in postmenopausal women: the quantity of available estrogens, the extent of 2-hydroxylation of parent estrogens, and the extent of methylation of 4-hydroxylation pathway catechols. Additional prospective studies will be required to test these specific findings and provide more information about circulating estrogens and estrogen metabolites in postmenopausal women, their associations with the risk of breast cancer, and the best models for describing those associations. If these biomarkers of estrogen metabolism are confirmed as predictors of breast cancer risk, they may become useful clinically and may suggest targets for preventive interventions.

\section{References}

1. Key T, Appleby P, Barnes I, Reeves G. Endogenous sex hormones and breast cancer in postmenopausal women: reanalysis of nine prospective studies. 7 Natl Cancer Inst. 2002;94(8):606-616.

2. Ziegler RG, Rossi SC, Fears TR, et al. Quantifying estrogen metabolism: an evaluation of the reproducibility and validity of enzyme immunoassays for 2-hydroxyestrone and 16alpha-hydroxyestrone in urine. Environ Health Perspect. 1997;105(suppl 3):607-614.

3. Adlercreutz H, Martin F. Biliary excretion and intestinal metabolism of progesterone and estrogens in man. I Steroid Biochem. 1980;13(2): 231-244.

4. Zhu BT, Han GZ, Shim JY, Wen Y, Jiang XR. Quantitative structureactivity relationship of various endogenous estrogen metabolites for human estrogen receptor alpha and beta subtypes: Insights into the structural determinants favoring a differential subtype binding. Endocrinology. 2006;147(9):4132-4150.

5. Stack DE, Byun J, Gross ML, Rogan EG, Cavalieri EL. Molecular characteristics of catechol estrogen quinones in reactions with deoxyribonucleosides. Chem Res Toxicol. 1996;9(5):851-859.

6. Liehr JG. Dual role of oestrogens as hormones and pro-carcinogens: tumour initiation by metabolic activation of oestrogens. Eur 7 Cancer Prev. 1997;6(1):3-10.
7. Chakravarti D, Mailander PC, Li KM, et al. Evidence that a burst of DNA depurination in SENCAR mouse skin induces error-prone repair and forms mutations in the H-ras gene. Oncogene. 2001;20(55):7945-7953.

8. Zahid M, Saeed M, Lu F, Gaikwad N, Rogan E, Cavalieri E. Inhibition of catechol-O-methyltransferase increases estrogen-DNA adduct formation. Free Radic Biol Med. 2007;43(11):1534-1540.

9. Raftogianis R, Creveling C, Weinshilboum R, Weisz J. Estrogen metabolism by conjugation. 7 Natl Cancer Inst Monogr. 2000;(27):113-124.

10. Michnovicz JJ, Hershcopf RJ, Naganuma H, Bradlow HL, Fishman J. Increased 2-hydroxylation of estradiol as a possible mechanism for the anti-estrogenic effect of cigarette smoking. N Engl 7 Med. 1986;315(21): 1305-1309.

11. Bradlow HL, Hershcopf R, Martucci C, Fishman J. 16 alpha-hydroxylation of estradiol: a possible risk marker for breast cancer. Ann N Y Acad Sci. 1986;464:138-151.

12. Cavalieri E, Rogan E. Catechol quinones of estrogens in the initiation of breast, prostate, and other human cancers: keynote lecture. Ann NY Acad Sci. 2006;1089:286-301.

13. Yager JD, Davidson NE. Estrogen carcinogenesis in breast cancer. N Engl 7 Med. 2006;354(3):270-282.

14. Stanczyk FZ, Clarke NJ. Advantages and challenges of mass spectrometry assays for steroid hormones. 7 Steroid Biochem Mol Biol. 2010;121(3-5): 491-495.

15. Faupel-Badger JM, Fuhrman BJ, Xu X, et al. Comparison of liquid chromatography-tandem mass spectrometry, RIA, and ELISA methods for measurement of urinary estrogens. Cancer Epidemiol Biomarkers Prev. 2010;19(1):292-300.

16. Xu X, Roman JM, Issaq HJ, et al. Quantitative measurement of endogenous estrogens and estrogen metabolites in human serum by liquid chromatography-tandem mass spectrometry. Anal Chem. 2007;79(20): 7813-7821.

17. Hayes RB, Sigurdson A, Moore L, et al. Methods for etiologic and early marker investigations in the PLCO trial. Mutat Res. 2005;592(1-2): $147-154$.

18. Lacey JV Jr., Kreimer AR, Buys SS, et al. Breast cancer epidemiology according to recognized breast cancer risk factors in the Prostate, Lung, Colorectal and Ovarian (PLCO) Cancer Screening Trial Cohort. BMC Cancer. 2009;9:84.

19. Chlebowski RT, Anderson GL, Gass M, et al. Estrogen plus progestin and breast cancer incidence and mortality in postmenopausal women. $7 A M A$. 2010;304(15):1684-1692.

20. Tworoger SS, Hankinson SE. Collection, processing, and storage of biological samples in epidemiologic studies: sex hormones, carotenoids, inflammatory markers, and proteomics as examples. Cancer Epidemiol Biomarkers Prev. 2006;15(9):1578-1581.

21. RTI, ed. SUDAAN User's Manual. Release. 8.0 ed. Research Triangle Park, NC: Research Triangle Institute; 2001.

22. Altekruse SF, Kosary CL, Krapcho M, et al., eds. SEER Cancer Statistics Review, 1975-2007. Bethesda, MD: National Cancer Institute. http://seer. cancer.gov/csr/1975_2007/.2010. Accessed April 9, 2010. Based on November 2009 SEER data submission, posted to the SEER web site.

23. Kono S, Merriam GR, Brandon DD, Loriaux DL, Lipsett MB, Fujino T. Radioimmunoassay and metabolic clearance rate of catecholestrogens, 2-hydroxyestrone and 2-hydroxyestradiol in man. 7 Steroid Biochem. 1983; 19(1B):627-633.

24. Kabat GC, Chang CJ, Sparano JA, et al. Urinary estrogen metabolites and breast cancer: a case-control study. Cancer Epidemiol Biomarkers Prev. 1997;6(7):505-509.

25. Ho GH, Luo XW, Ji CY, Foo SC, Ng EH. Urinary 2/16 alphahydroxyestrone ratio: correlation with serum insulin-like growth factor binding protein-3 and a potential biomarker of breast cancer risk. Ann Acad Med Singapore. 1998;27(2):294-299.

26. Ursin G, London S, Stanczyk FZ, et al. Urinary 2-hydroxyestrone/16alphahydroxyestrone ratio and risk of breast cancer in postmenopausal women. f Natl Cancer Inst. 1999;91(12):1067-1072.

27. Fowke JH, Qi D, Bradlow HL, et al. Urinary estrogen metabolites and breast cancer: differential pattern of risk found with pre- versus posttreatment collection. Steroids. 2003;68(1):65-72. 
28. Kabat GC, O'Leary ES, Gammon MD, et al. Estrogen metabolism and breast cancer. Epidemiology. 2006;17(1):80-88.

29. Meilahn EN, De Stavola B, Allen DS, et al. Do urinary oestrogen metabolites predict breast cancer? Guernsey III cohort follow-up. Br 7 Cancer. 1998;78(9):1250-1255.

30. Muti P, Bradlow HL, Micheli A, et al. Estrogen metabolism and risk of breast cancer: a prospective study of the 2:16alpha-hydroxyestrone ratio in premenopausal and postmenopausal women. Epidemiology. 2000;11(6): 635-640.

31. Cauley JA, Zmuda JM, Danielson ME, et al. Estrogen metabolites and the risk of breast cancer in older women. Epidemiology. 2003;14(6):740-744.

32. Wellejus A, Olsen A, Tjonneland A, Thomsen BL, Overvad K, Loft S. Urinary hydroxyestrogens and breast cancer risk among postmenopausal women: a prospective study. Cancer Epidemiol Biomarkers Prev. 2005;14(9): 2137-2142.

33. Modugno F, Kip KE, Cochrane B, et al. Obesity, hormone therapy, estrogen metabolism and risk of postmenopausal breast cancer. Int 7 Cancer. 2006;118(5):1292-1301.

34. Eliassen AH, Missmer SA, Tworoger SS, Hankinson SE. Circulating 2-hydroxy- and 16alpha-hydroxy estrone levels and risk of breast cancer among postmenopausal women. Cancer Epidemiol Biomarkers Prev. 2008; 17(8):2029-2035.

35. Arslan AA, Shore RE, Afanasyeva Y, Koenig KL, Toniolo P, ZeleniuchJacquotte A. Circulating estrogen metabolites and risk for breast cancer in premenopausal women. Cancer Epidemiol Biomarkers Prev. 2009;18(8): 2273-2279.

36. Falk RT, Rossi SC, Fears TR, et al. A new ELISA kit for measuring urinary 2-hydroxyestrone, 16alpha-hydroxyestrone, and their ratio: reproducibility, validity, and assay performance after freeze-thaw cycling and preservation by boric acid. Cancer Epidemiol Biomarkers Prev. 2000;9(1): 81-87.

37. Bransfield LA, Rennie A, Visvanathan K, et al. Formation of two novel estrogen guanine adducts and HPLC/MS detection of 4-hydroxyestradiolN7-guanine in human urine. Chem Res Toxicol. 2008;21(8):1622-1630.

38. Liehr JG, Avitts TA, Randerath E, Randerath K. Estrogen-induced endogenous DNA adduction: possible mechanism of hormonal cancer. Proc Natl Acad Sci USA. 1986;83(14):5301-5305.

39. Zhu BT. Catechol-O-Methyltransferase (COMT)-mediated methylation metabolism of endogenous bioactive catechols and modulation by endobiotics and xenobiotics: importance in pathophysiology and pathogenesis. Curr Drug Metab. 2002;3(3):321-349.

40. Mailander PC, Meza JL, Higginbotham S, Chakravarti D. Induction of A.T to G.C mutations by erroneous repair of depurinated DNA following estrogen treatment of the mammary gland of ACI rats. F Steroid Biochem Mol Biol. 2006;101(4-5):204-215.

41. Liehr JG, Fang WF, Sirbasku DA, Ari-Ulubelen A. Carcinogenicity of catechol estrogens in Syrian hamsters. I Steroid Biochem. 1986;24(1): 353-356.

42. Cavalieri EL, Stack DE, Devanesan PD, et al. Molecular origin of cancer: catechol estrogen-3,4-quinones as endogenous tumor initiators. Proc Natl Acad Sci U S A. 1997;94(20):10937-10942.
43. Zhao Z, Kosinska W, Khmelnitsky M, et al. Mutagenic activity of 4-hydroxyestradiol, but not 2-hydroxyestradiol, in BB rat2 embryonic cells, and the mutational spectrum of 4-hydroxyestradiol. Chem Res Toxicol. 2006;19(3):475-479.

44. Crooke PS, Ritchie MD, Hachey DL, Dawling S, Roodi N, Parl FF. Estrogens, enzyme variants, and breast cancer: a risk model. Cancer Epidemiol Biomarkers Prev. 2006;15(9):1620-1629.

45. Canzian F, Cox DG, Setiawan VW, et al. Comprehensive analysis of common genetic variation in 61 genes related to steroid hormone and insulin-like growth factor-I metabolism and breast cancer risk in the NCI breast and prostate cancer cohort consortium. Hum Mol Genet. 2010;19(19):3873-3884.

\section{Funding}

This work was supported by the Intramural Research Programs of the Division of Cancer Epidemiology and Genetics and the Center for Cancer Research of the National Cancer Institute (NCI), National Institutes of Health (NIH); the Division of Cancer Prevention of the NCI, NIH; and contract HHSN261200800001E to SAIC, Inc, from NCI, NIH, DHHS.

\section{Notes}

The authors thank Dr Philip Prorok, Division of Cancer Prevention, National Cancer Institute; the Screening Center investigators and staff of the Prostate, Lung, Colorectal, and Ovarian Cancer Screening Trial (PLCO); Mr Tom Riley and staff, Information Management Services, Inc; Ms. Barbara O'Brien and staff, Westat, Inc; Mr Tim Sheehy and staff, DNA Extraction and Staging Laboratory, SAIC-Frederick, Inc; and Ms Jackie King and staff, BioReliance Corporation. Most importantly, we acknowledge the study participants for their contributions to making this study possible. We would also like to thank Messrs Michael Stagner, David Check, and Micah Ziegler for assistance with article figures. L. K. Keefer, T. D. Veenstra, X. Xu, and R. G. Ziegler receive royalties from the National Institutes of Health for the liquid chromatography-tandem mass spectrometry assay used in this study, which was developed and patented at the National Cancer Institute. The authors are solely responsible for the study design, data collection and analysis, interpretation of the data, and the preparation of the article.

Affiliations of authors: Epidemiology and Biostatistics Program, Division of Cancer Epidemiology and Genetics, National Cancer Institute, Bethesda, MD (BJF, CS, MHG, LYS, RNH, RGZ); Information Management Services, Inc, Silver Spring, MD (JB-M); Laboratory of Proteomics and Analytical Technologies, Advanced Technology Program, SAIC-Frederick, Inc, National Cancer Institute at Frederick, Frederick, MD (XX, TDV); Internal Medicine, University of Utah Health Sciences Center, Salt Lake City, UT (SSB); Department of Medicine and Oncology, Lombardi Cancer Center, Georgetown University, Washington, DC (Cl); Laboratory of Comparative Carcinogenesis, Center for Cancer Research, National Cancer Institute at Frederick, Frederick, MD (LKK); Early Detection Research Group, Division of Cancer Prevention, National Cancer Institute, Bethesda, $\mathrm{MD}(\mathrm{CDB})$. 